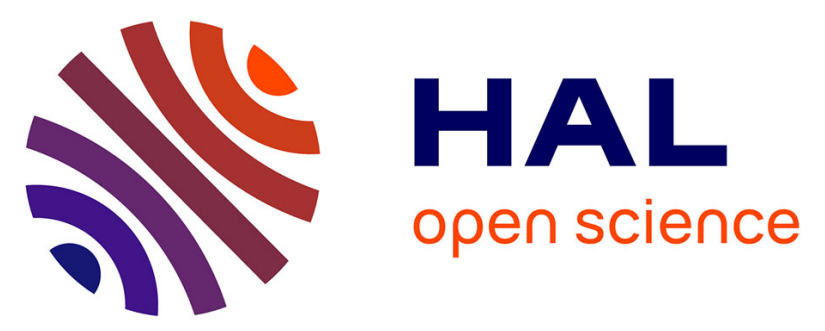

\title{
Netted Multi-Function Radars Positioning and Modes Selection by Non-Holonomic Fast Marching Computation of Highest Threatening Trajectories \& by CMA-ES Optimization
}

Johann Dreo, François Desquilbet, Frederic Barbaresco, Jean-Marie Mirebeau

\section{To cite this version:}

Johann Dreo, François Desquilbet, Frederic Barbaresco, Jean-Marie Mirebeau. Netted Multi-Function Radars Positioning and Modes Selection by Non-Holonomic Fast Marching Computation of Highest Threatening Trajectories \& by CMA-ES Optimization. 2019 International Radar Conference (RADAR), Sep 2019, TOULON, France. pp.1-6, 10.1109/RADAR41533.2019.171285 . hal-03030426

\author{
HAL Id: hal-03030426 \\ https://hal.science/hal-03030426
}

Submitted on 3 Dec 2020

HAL is a multi-disciplinary open access archive for the deposit and dissemination of scientific research documents, whether they are published or not. The documents may come from teaching and research institutions in France or abroad, or from public or private research centers.
L'archive ouverte pluridisciplinaire HAL, est destinée au dépôt et à la diffusion de documents scientifiques de niveau recherche, publiés ou non, émanant des établissements d'enseignement et de recherche français ou étrangers, des laboratoires publics ou privés. 


\section{Netted Multi-Function Radars Positioning and Modes Selection by Non-Holonomic Fast Marching Computation of Highest Threatening Trajectories \& by CMA-ES Optimization}

\author{
Johann DREO \\ Thales Research \& Technology \\ Palaiseau, FRANCE \\ johann.dreo@thalesgroup.com \\ Frédéric BARBARESCO \\ Thales Land \& Air Systems \\ Limours, FRANCE \\ frederic.barbaresco@thalesgroup.com
}

\author{
François DESQUILBET \\ Département de Mathématiques et Applications \\ École Normale Supérieure (ENS Ulm) \\ Paris, FRANCE \\ francois.desquilbet@ens.fr \\ Jean-Marie MIREBEAU \\ Laboratoire de Mathématiques d'Orsay, \\ Université Paris-Sud, CNRS, Université Paris-Saclay \\ Orsay, FRANCE \\ jean-marie.mirebeau@math.u-psud.fr
}

\begin{abstract}
We aim at designing a radar network which maximizes the detection probability of the worst threatening trajectory which can reach a protected area. In game theory, we represent this problem as a non-cooperative zero-sum game: a first player chooses a setting for the network, and the other player chooses a trajectory from the admissible class of trajectories with full information over the network. The players' objective are respectively to maximize and minimize the path cost which is the detection probability integrated along the trajectory in the network. In comparison with previous works, we added ambiguity maps depending on the distance and the radial speed, which are functions of internal parameters of the radars that can be optimized: PRI (Pulse Repetition Interval) and frequency. We also take into account RCS (Radar Cross Section) and a complex geometry with masks deduced from the DEM (Digital Elevation Map) and Earth curvature. The computation of optimal trajectories is performed by a specialized variant of the Fast-Marching algorithm, devoted to computing curves that globally minimize an energy featuring both a data driven term and a second order curvature penalizing term. The profile of the cost function with regard to the direction of movement is nonconvex, which is significant only with a curvature penalization: we chose the Dubins model, in which the curvature radius is bounded. We illustrate results on different Use-Cases.
\end{abstract}

Keywords - Anisotropic Eikonal Equation, Fast Marching, Multifunction Radar

\section{THREATENING TRAJECTORIES MITIGATION FOR A NETWORK OF RADARS}

We optimize the configuration of a radar network protecting an area, against an enemy assumed to have unlimited intelligence and computing power, and yet whose vehicle is subject to some maneuverability constraints. The goal is to maximize the probability of detection of the most dangerous trajectory integrated along its path between a given origin and a place to protect, which will take advantage of any hideout in the terrain, blind spot or physical limitation in the radar network. The trajectory is only subject to a lower bound in the turning radius, due to the vehicle high speed. We model this problem as a non-cooperative zero-sum game: a first player chooses a setting $\xi$ for the radar detection network $\aleph$, and the other player chooses a trajectory $\gamma$ from the admissible class $\Gamma$ with full information over the network. The players' objective is respectively to maximize and minimize the path cost:

$$
C(\aleph, \Gamma)=\operatorname{supinf}_{\xi \in \aleph} \Theta_{\xi \in \Gamma}(\gamma)
$$

where $\Theta_{\xi}(\gamma)$ is the detection probability (integrated along the path) of the trajectory $\gamma$ in the network $\xi$. Minimization over $\gamma \in \Gamma$ (given $\xi \in \aleph$ ) is performed using the fast and reliable techniques of $\S I$ II. We rely on the CMA-ES algorithm for the subsequent optimization over $\xi \in \aleph$, which is rather difficult (non-convex, nondifferentiable).

In comparison with earlier works [3], we use the curvature bounded Dubins model to reject non-physical enemy trajectories, featuring e.g. angular turns or oscillations in the vehicle direction. We also considerably improve, relative to [1], the detection probability model, used to define $\Theta_{\xi}(\gamma)$, taking into account the three following factors respectively related to the radar, the target, and the terrain.

- The ambiguity map accounts for the probability of detection of a generic target by a radar, depending on the distance and the radial speed of the target relatively to the radar, see Figure 1. There are blind speed areas, due to sampling repetition interval and pulse duration causing blind radial distances and blind radial speeds. The positions of the blind areas are periodical and depend on internal parameters of the radar that can be optimized: signal wavelength, and pulse repetition interval.

- The radar cross section accounts for the probability of detection of a specific target, depending on its orientation relatively to the radar (Figure 2). For instance, a furtive plane often has a low probability of detection if seen from the front, and a higher one if seen 
from the side. In our case, we used a simple model with a dependency on frequency, but a more complex model is possible.

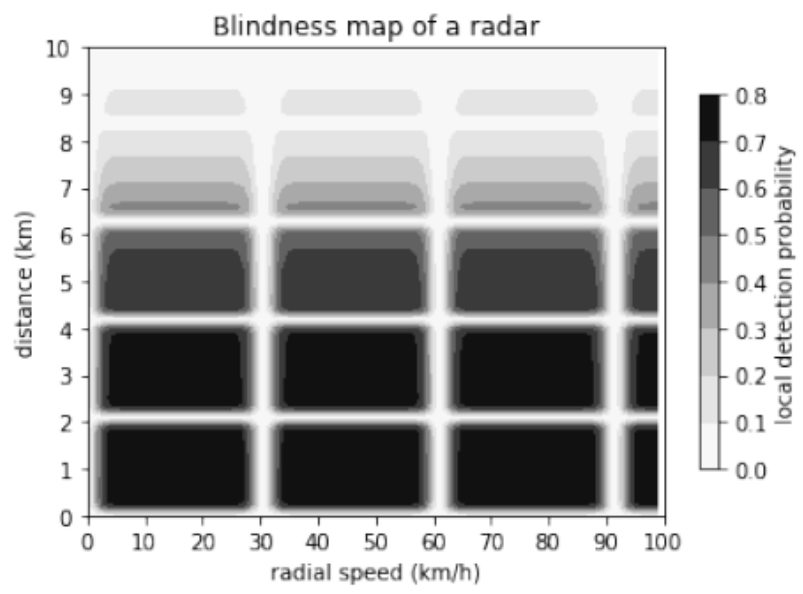

Fig. 1. Ambiguity map for a selected waveform waveform

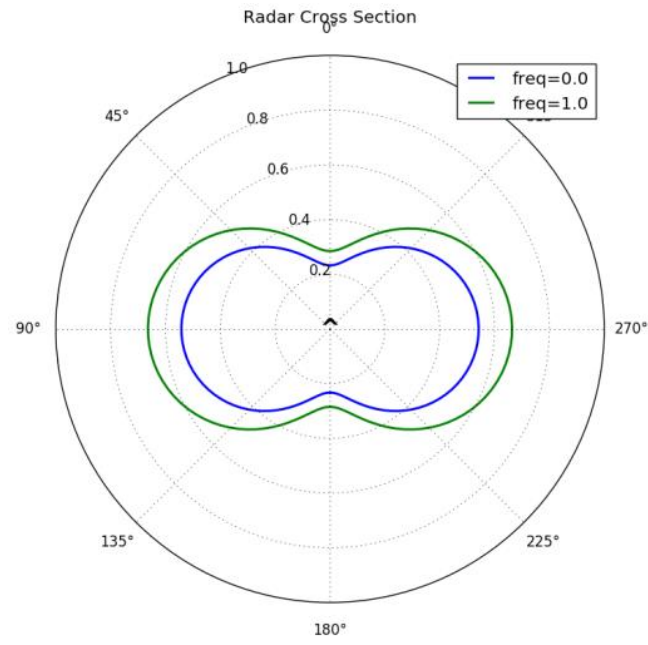

Fig. 2. Anisotropy of Radar Cross Section

- The elevation map is used to determine blind regions in the terrain due to obstruction of the radar line of sight. In a mountainous area, a target can take advantage of valleys to move "under the Radar coverage". The Earth curvature is also taken into account.

The profile of the cost function with regard to the direction of movement is typically non-convex, which is significant only in the presence of a curvature penalization. For that, we choose the Dubins model, in which the curvature radius is bounded. We showcase the following three phenomena:

- Trajectories dodging radars through their blind distances (cf. Figure 3.). In this picture, only the positional factor in the cost map is shown in greyscale, and not the part of the cost depending on the orientation. The red line represents the optimal trajectory of the target, going from the left to the right of a rectangular domain, with a radar in the center. It features a circle arc, at a precise blind distance from the radar, and two spiral arcs.

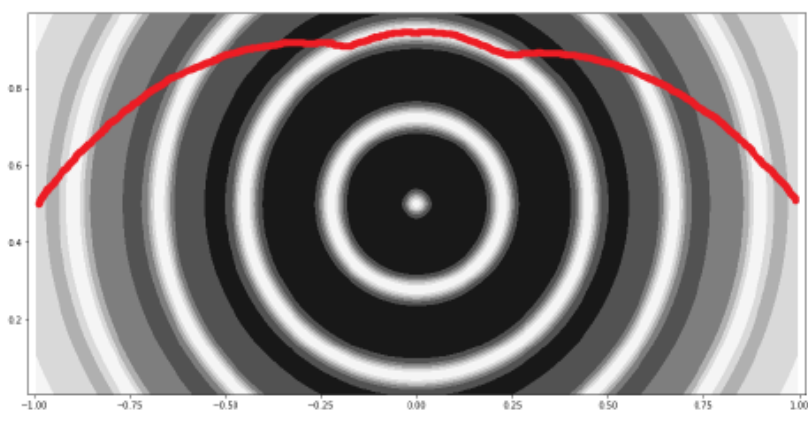

Fig. 3. Dodging a radar through a blind distance

- Spiraling threatening trajectories, taking advantage of the blind radial speed (cf. Figure 4). The red line represents the trajectory of the target, going from the left to the center of the domain where the radar is located, maintaining a constant angle with the radar in order to minimize visibility, except at the end due to the imposed bound on path curvature. Figure 5 shows the same spiral, along with the "zigzag" trajectory that would be optimal if no curvature constraint was taken into account.

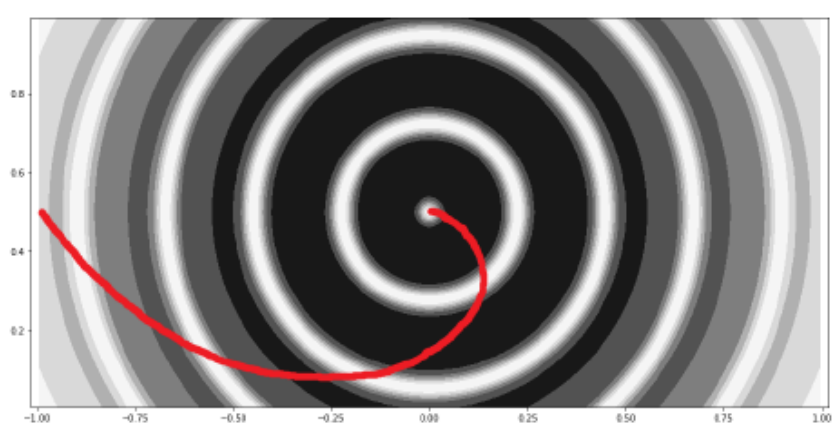

Fig. 4. Spiraling threatening trajectory

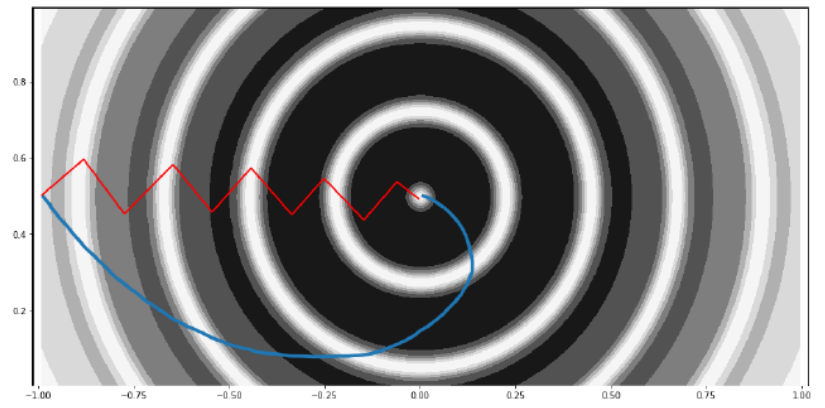

Fig. 5. In red, Degenerated behaviour with no penalization of curvature, and in blue with curvature constrained solution

- Hiding in valleys. A digital elevation map, of $50 \mathrm{~km} \times 50 \mathrm{~km}$ in a mountain area, is used to construct a probability of detection map (cf. Figure 6). Threatening trajectories tend to concentrate in valleys. The optimized radar positions a re close to the target to be defended, and either on high ground or in alignment with long valleys. 


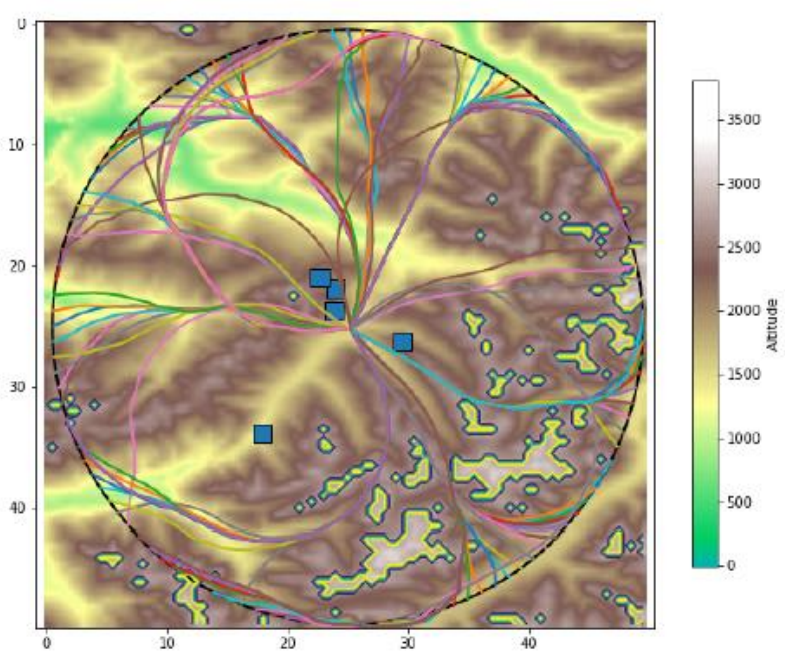

Fig. 6. Threatening trajectories, from a circular region towards its center point, with optimized radar positions, and digital elevation map

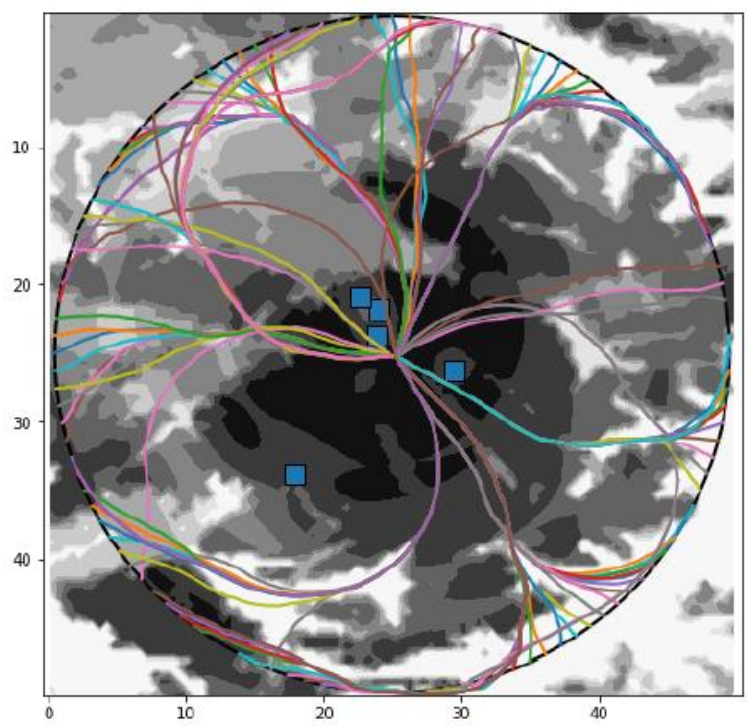

Fig. 7. Positional factor in the cost map

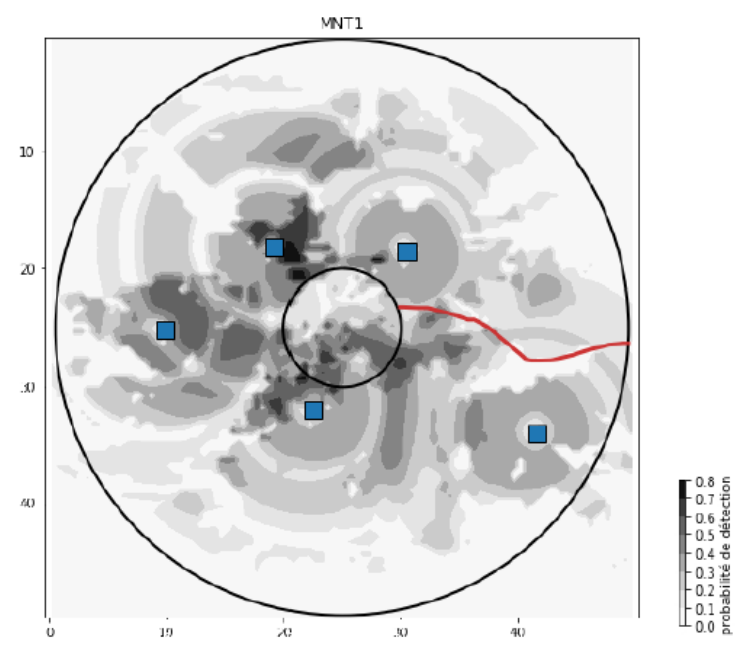

Fig. 8. Most threatening trajectories from 1 pont on the limit of coverage to a radial distance from the site to be protected (with cost map in the back ground)

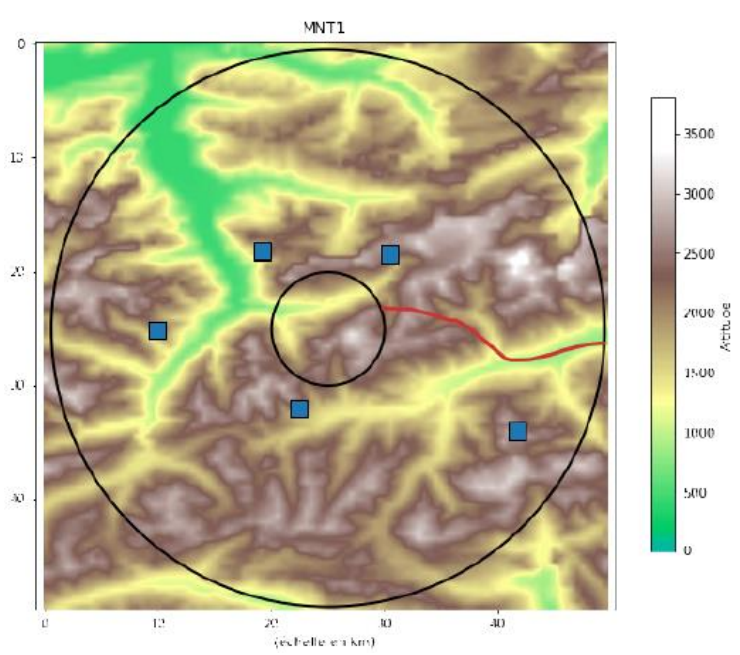

Fig. 9. Most threatening trajectories from 1 pont on the limit of coverage to a radial distance from the site to be protected (with DEM in the background)

\section{GLOBALLY OPTIMAL PATHS WITH A CURVATURE PENALTY}

This paper deals with planar paths minimizing a specific energy functional, between two given points and with prescribed tangents at these points. The path energy model features a low order data-driven term, and a higher order regularization term. A globally optimal path is found, using optimal control techniques, which involve numerically solving a PDE on the configuration space of positions and orientations.

\section{A. Path energy models}

In the models of interest to us, the cost of a smooth planar path $x:[0, T] \rightarrow \Omega$, parametrized by Euclidean arc length and within a domain $\Omega \subset \square^{2}$, takes the following form:

$\Theta(x)=\int_{0}^{T} \alpha(x(s), \dot{x}(s)) C(\|\ddot{x}(s)\|) d s$

We denoted by $\left.\alpha: \bar{\Omega} \times S^{1} \rightarrow\right] 0,+\infty[$ an arbitrary continuous data-driven term, depending on the path position and direction. The path local curvature $\kappa=\|\ddot{x}(t)\|$ (recall that $\|\dot{x}(s)\| \equiv 1$ ) is penalized in (1) by a cost function $C(\kappa)$, which may be chosen among the following classical models, here sorted by increasingly stiffness:

- Reeds-Shepp: $\sqrt{1+\kappa^{2}}$

- $\quad$ Euler-Mumford: $1+\kappa^{2}$

- Dubins: $\left\{\begin{array}{l}1 \text { if } \kappa \leq 1 \\ \infty \text { else }\end{array}\right.$

They are respectively representative of (i) a wheelchair-like robot, (ii) the bending energy of an elastic bar, and (iii) a vehicle with a bounded turning radius. In the case of the Reeds-Shepp model, one must further distinguish between 
the classical model with reverse gear, and the forward only variant.

\section{B. Viscosity solutions, and the Fast marching algorithm}

Data-driven path energies, subject to e.g. fixed endpoints, usually possess many local minima. In order to guarantee that the global minimum is found, path energy minimization must be reformulated as an optimal control problem. The corresponding value function is the unique viscosity solution to a PDE of eikonal type, and the optimal paths can be extracted by backtracking once it is numerically computed.

Only simple first order energies, such as defined by $\int_{0}^{T} \alpha(x(s))\|\dot{x}(s)\| d s$ could originally be addressed in the viscosity solution framework, typically using the Fast Marching Method (FMM) which solves the eikonal PDE in a single pass over the domain. Recent progress [2] enabled the Anisotropic Fast Marching, in order to solve (1). For that purpose the path is lifted in the configuration space of positions and orientations, defining $\gamma(t)=(x(t), \theta(t))$ subject to the constraint $x^{\prime}(t)=(\cos \theta(t), \sin \theta(t))$. This allows to reformulate (1) as a first order energy, since $\left|x^{\prime \prime}(t)\right|=\left|\theta^{\prime}(t)\right|$. See [2] for details and comparison with alternative approaches.

\section{OPTIMIZATION SCENARIO}

Digital elevation map is shown in Figure 10, in a domain of $50 \mathrm{~km} \times 50 \mathrm{~km}$. The radar coverage is however complex in the area, due to the high relief from the mountains. The target could fly undetected at a low altitude by hiding in valleys, see Figure 11. The trajectories are computed from a regular sample of the circular boundary of the domain, toward a circular boundary close to the point of interest (cf. Figures $8 \& 9$ ). The optimized objective function is the smallest probability of detection among all the optimal trajectories reaching the close boundary (cf. Figure $8 \& 9$ ), given a configuration of the radar network.

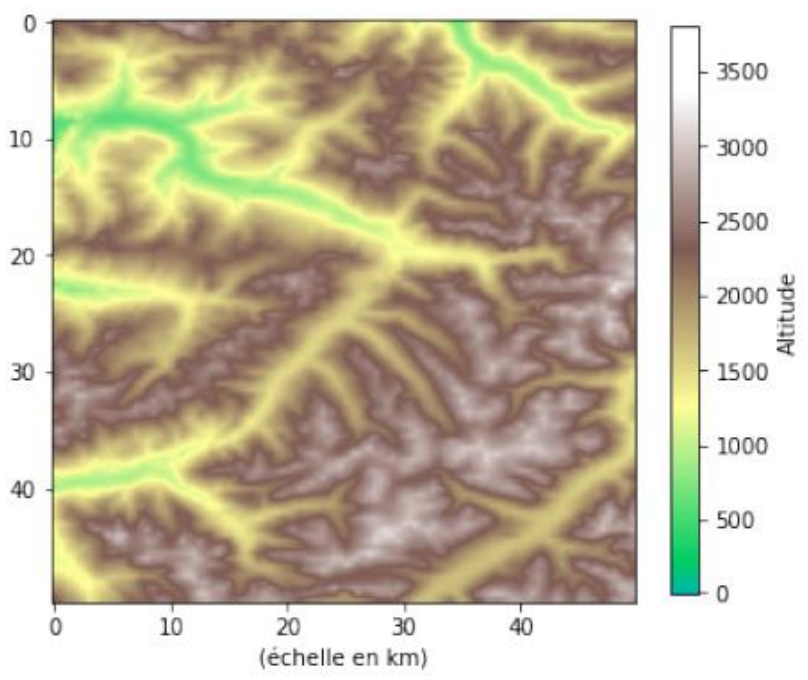

Fig. 10. Digital Elevation Map of the Use-Case in Moutain Area

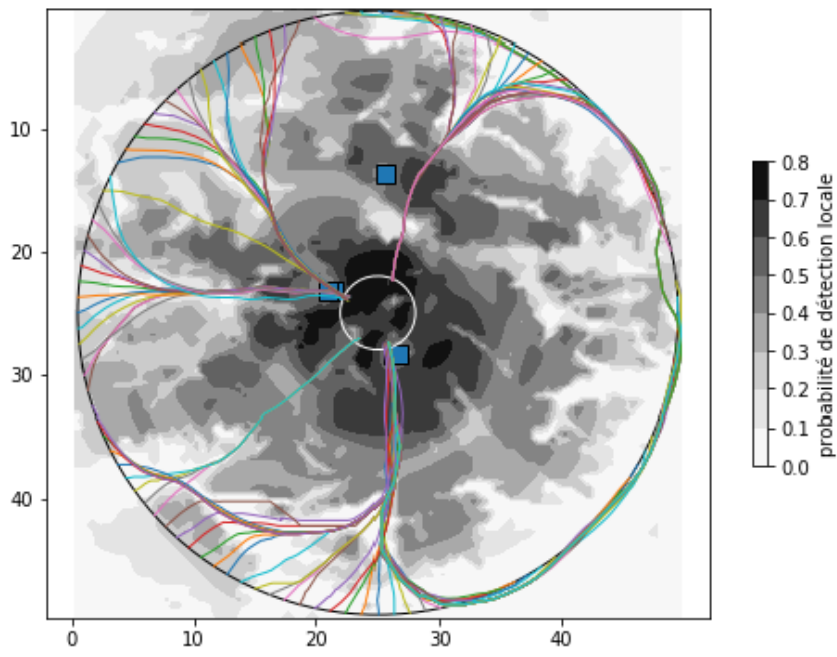

Fig. 11. Threatening trajectories in a non-optimized network

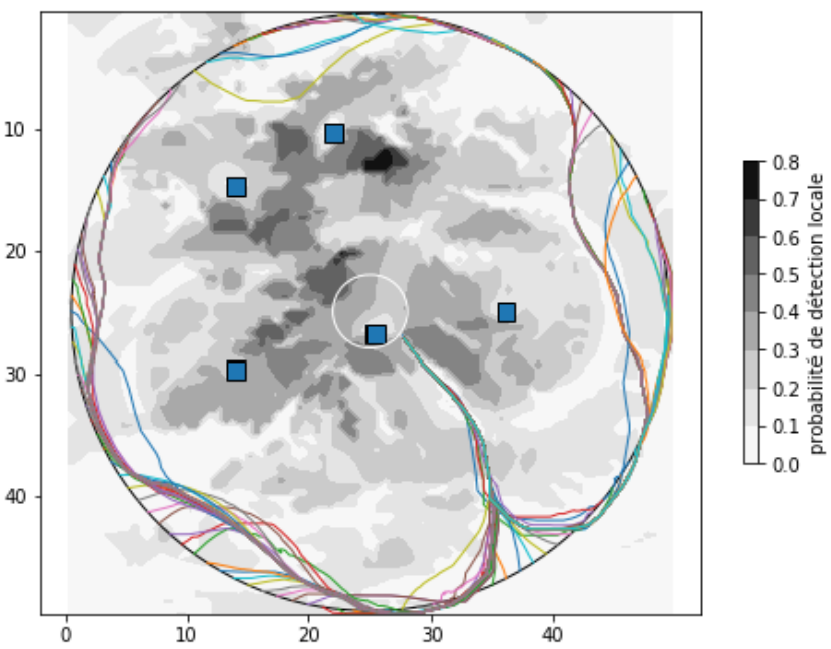

Fig. 12. Threatening trajectories in an optimized network

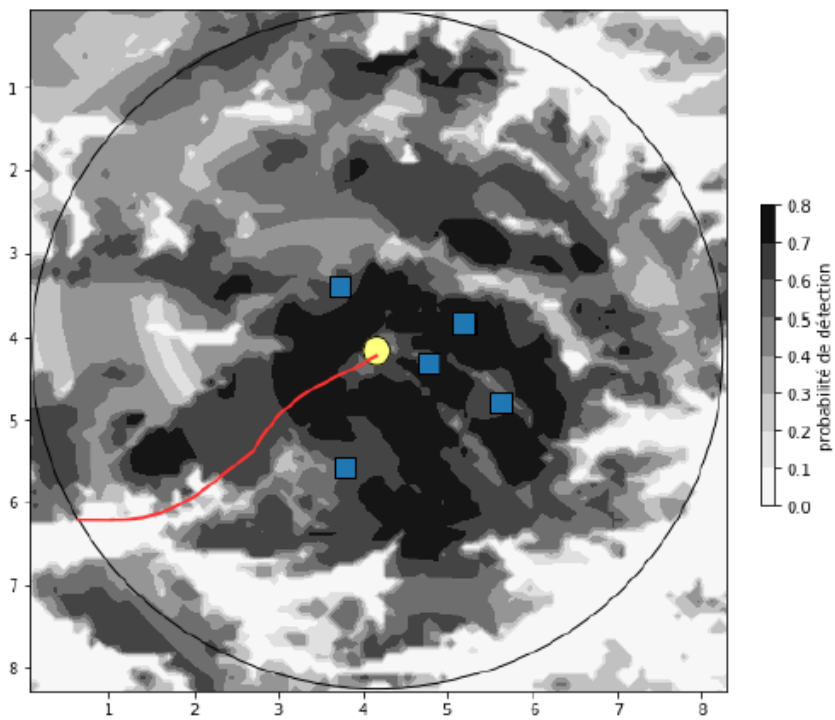

Fig. 13. Radars Positions Optimisation in blue, with highest threatening trajectory in red. 


\section{CMA-ES OPTIMIZATION ALGORITHM}

The CMA-ES algorithm is one of the most powerful stochastic numerical optimizers to address difficult blackbox problems. Its intrinsic time and space complexity is quadratic limiting its applicability with increasing problem dimensionality. To circumvent this limitation, different large-scale variants of CMA-ES with sub-quadratic complexity have been proposed [11].

For solving black-box optimization problem of this usecase, we have used the CMA-ES algorithm developed at Paris-Saclay University. The performance of this algorithm on our problem is estimated via ERT-ECDF (expected running time empirical cumulative density function): over a large number of runs, calculation of the probability to have a set margin of error, depending on the number of function calls (Figure 14). Difference in threats between a bad configuration and the best one are shown on Figure 11, 12 and 13 .

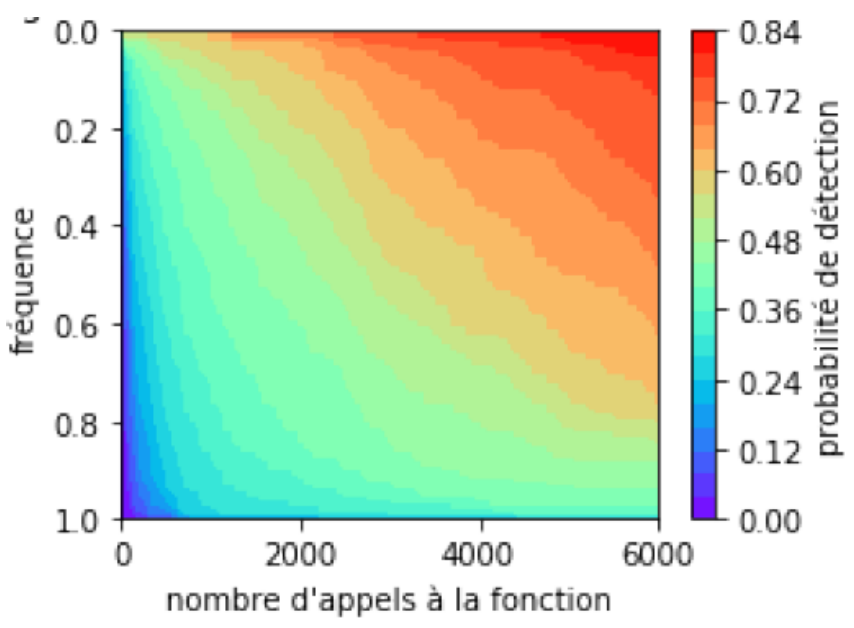

Fig. 14. ERT for all scenarios with 150 runs of CMA-ES algorithm

For our Use-Case, 20 parameters have been optimized: 4 parameters for each of the radars (position $(x, y)$, Pulse Repetition Interval, wavelength). The trajectory search space has been discretized in the domains: $100 \times 100$ for spatial variables, 120 angles for the angular variable. Estimated Computation time for this Use-Case is of a few seconds per function call (largely dominated by the computation of the highest threatening trajectory for a configuration of the radars). The number of function calls needed to find a configuration with a detection probability of $50 \%$ in $90 \%$ of runs is close to 6000 (around a day of computation), as shown on Figure 14.

\section{CONCLUSION}

We have proved robustness of new algorithms for optimal configuration of netted radars, to reduce integrated probability of detection along the highest threatening trajectory. We have illustrated with realistic modelling of generic anistropic cost functions: ambiguity map, radar cross-section, digital elevation map... AFM (Anisotropic Fast Marching) algorithm can take into account a penalization of curvature in the computation of shortest path, which is critical in that setting.

Other uses cases for the AFM algorithm could be elaborated:

- configuration of a radar who lost a target,

- use of mobile radars,

- adding passive radars to cover the defects of the network.

Future works will be devoted to further enhancing the model, taking into account limited knowledge of the ennemy (e.g. due to the use of passive radar receivers), introducing success criteria more complex than mere detection (e.g. requiring detection early enough for interception), and adapting optimization solvers to the problem in order to reach betterperformances.

\section{REFERENCES}

[1] J-M. Mirebeau, J. Dreo. Automatic differentiation of non-holonomic fast marching for computing most threatening trajectories under sensors surveillance. In : Nielsen, F., Barbaresco, F. (eds.) Proceedings of Third International Conference on Geometric Science of Information (GSI). Paris, 2017.

[2] J-M. Mirebeau. Fast-marching methods for curvature penalized shortest paths. Journal of Mathematical Imaging and Vision, 2018.

[3] F. Barbaresco. Computation of most threatening radar trajectories areas and corridors based on fast-marching \& Level Sets. In IEEE Symposium On Computational Intelligence For Security And Defence Applications, pages 51-58. IEEE, 2011.

[4] J-M. Mirebeau, Jorg Portegies. Hamiltonian Fast Marching : A numerical solver for anisotropic and non-holonomic eikonal PDEs. 2018

[5] J-M. Mirebeau. Numerical schemes for anisotropic pdes on cartesian domains. Diplôme d'habilitation à diriger les recherches de l'Université Paris-Sud, 2018.

[6] R. Cariou. Limitation de la détectabilité d'un avion dans un environnement électromagnétique hostile par optimisation de sa trajectoire. Thèse DGA MI, 16 april 2013

[7] M. Bardi, I. Capuzzo-Dolcetta. Optimal control and viscosity solutions of Hamilton-Jacobi-Bellman equations. Springer Science \& Business Media, 2008.

[8] J-M. Mirebeau. Github Repository, https ://github.com/Mirebeau.

[9] G Gauguelin and F. Barbaresco, "Optimal and most threatening trajectories estimation based on Eikonal equation with local geometric constraint", COGIS 2007, Stanford University, 2007

[10] J.P. Zolesio, "Set weak evolution and transverse field, Variation application and shape derivation", INRIA Report no 4649, 2002

[11] K. Varelas, A. Auger, D. Brockhoff, N. Hansen, O. Elhara, Y. Semet, R. Kassab, F. Barbaresco, Comparative Study of Large-scale Variants of CMA-ES, PPSN XV 2018 - 15th International Conference on Parallel Problem Solving from Nature, Sep 2018, Coimbra, Portugal. 11101,pp.3-15, LNCS 